\title{
Gene polymorphisms are associated with clinical outcome in Chinese resected laryngeal carcinoma patients
}

\author{
Peng Chen ${ }^{1,2,3, *}$, Zhengshuai Chen ${ }^{1,2, *}$, Jinglie Li $^{1,2}$, Hua Yang ${ }^{1,2}$, Yuanyuan Zhu ${ }^{2}$, \\ Ning Zhang ${ }^{2}$, Mengdan Yan ${ }^{1,2}$, Yuan Shao ${ }^{4}$, Chao Chen ${ }^{1,2}$, Tianbo Jin ${ }^{1,2}$ \\ ${ }^{1}$ The National Engineering Research Centre for Miniaturized Detection Systems, College of Life Science, Northwest University, \\ Xi'an, Shaanxi 710069, China \\ ${ }^{2}$ Key Laboratory of Resource Biology and Biotechnology in Western China (Northwest University), Ministry of Education, \\ Xi'an, Shaanxi 710069, China \\ ${ }^{3}$ Institution of Basic Medical Science, Xi'an Medical University, Xi'an, Shaanxi 710021, China \\ ${ }^{4}$ Department of Otolaryngology-Head and Neck Surgery, The First Affiliated Hospital of Xi'an, Jiaotong University, Xi'an, \\ Shaanxi 710061, China \\ *Joint first authors \\ Correspondence to: Tianbo Jin, email: jintianbo@gmail.com \\ Chao Chen, email: chaochenxd898@gmail.com
}

Keywords: single-nucleotide polymorphism, laryngeal carcinoma, overall survival, prognosis, prognostic marker

Received: July 25, 2016

Accepted: September 22, 2016

Published: September 28, 2016

\section{ABSTRACT}

We examined the multigenetic index on the progression of laryngeal carcinoma in Chinese population. This study aims to assess the effects of single nucleotide polymorphisms (SNPs) on survival of Laryngeal Carcinoma (LC) patients. Eighteen SNPs were selected and genotyped using the Sequenom iPLEX genotyping system in a cohort of 170 resected Chinese LC patients. Multivariate Cox proportional hazards model and Kaplan-Meier curve were used for the prognosis analysis. Overall, the median survival time (MST) was $\mathbf{3 8 . 0 0}$ months. The one, three and five year KaplanMeier survival rate was $0.847 \pm 0.028,0.572 \pm 0.038$ and $0.471 \pm 0.041$ respectively. The risks of death with the Hazard Ratio (HR) [95\% confidence intervals] (CI) of 2.40 (1.15-4.50), 2.17 (1.45-3.25), 2.39 (1.58-3.62), 3.29 (2.10-5.18), respectively. There was significant associations between the SNPs and OS when the entire study population was examined. The rs1321311 TG genotype (vs.GG), rs2494938 AA genotype (vs. GG) and rs9363918 TG genotype (vs. GG) were associated with a worse prognosis for OS (adjusted $H R=1.64 ; 95 \%$ confidence interval $=1.07-2.51$; $P=0.022$, adjusted $H R=2.85 ; P=0.12 ;$ adjusted $H R=1.78 ; P=0.009 ;$ respectively).

The results suggest for the first time that these gene polymorphisms may serve as an independent prognostic marker for LC patients.

\section{INTRODUCTION}

Laryngeal carcinoma (LC) is one of the most frequently diagnosed malignant tumors in the Department of Otolaryngology - Head and Neck Surgery at the First Affiliated Hospital of Xi'an Jiaotong University [1]. LC can spread by directly invading adjacent structures, by metastasizing to regional cervical lymph nodes, or through the blood stream to more distant locations; among these, distant metastases in the lungs are the most common $[2,3]$. LC resulted in 88,000 deaths in 2013 , up from 76,000 deaths in 1990. Local recurrence after treatment is common, occurring in 10 to $50 \%$ of patients, and five-year survival rates in the United States are 60\%. Adverse clinical prognostic factors in LC include advanced disease stage and poor performance status. However, clinical factors often do not accurately predict LC outcomes, and although a number of molecular markers accurately predict overall survival, none are currently used in the clinical setting [4].

The importance of genetic alterations in cancer has long been recognized, and although LC prognosis is not dependent on a single gene, it is affected by genetic differences between populations. The novel gene polymorphisms identified here that are associated with clinical outcomes in LC patients may help to improve personalized treatment strategies. Our findings suggest 
that these genes may be useful when screening for LC in Han Chinese populations and might aid in the discovery of additional molecular mechanisms that contribute to LC. [5] and Zhang et al.[6] were Genome scanningand PCR-RFLP methods have revealed that the H-RAS T81C mutation is associated with increased susceptibility to sporadic colorectal cancer. Mutations in P53, a human tumor suppressor gene, are involved in at least $1 / 2$ of all tumors [7]. Single-nucleotide mutations are the most common genetic variations in the human genome, and the frequency of individual SNPs varies among different populations. Studying SNPs may therefore help explain individual and intra-population differences in susceptibility to complex diseases, drug resistance, and response to environmental factors. Recently, several separate genome-wide association scans have demonstrated that common SNPs, including those in the leucine rich repeat and fibronectin type III domain containing 2 (LRFN2), cyclin-dependent kinase inhibitor 1A (CDKN1A), brain-specific angiogenesis inhibitor 3 (BAI3), and parkin RBR E3 ubiquitin protein ligase (PARK2) genes, are associated with LC prognosis. All of these genes are located on Chromosome 5, 6, or 7 [8-10].

SNPs, which can serve as surrogates for patient's general genetic backgrounds, can predict therapeutic responses and prognoses [11, 12]. However, whether these variations influence clinical outcomes in LC patients remains unclear, and little is known regarding these associations specifically in Chinese patients. In this study, we assessed the association of 11 functional SNPs with survival in a cohort of 170 Chinese resected LC patients. To the best of our knowledge, this is the first study to investigate the predictive role of these SNPs for LC prognosis.

\section{RESULTS}

\section{Patient characteristics and prognosis analysis}

Between January 2002 and April 2013, a total of 170 laryngeal carcinoma patients (104 who underwent partial laryngectomy and 66 who underwent total laryngectomy) were included in this study. Patient characteristics are listed in Table 1 and Table S1. The median age was 60.75 years (range, 32 to 82 years). Of the 170 patients, $21.8 \%$ had stage I, $21.2 \%$ had stage II, $35.9 \%$ had stage III, and $21.2 \%$ had stage IV disease at the time of diagnosis. The median follow-up time was 38.00 months (range, 3 to 122 months). Of the 170 patients, 100 died before the final follow-up. Overall, the median survival time (MST) was 38.00 months, and the one, three, and five year KaplanMeier survival rates were $0.847 \pm 0.028,0.572 \pm 0.038$, and $0.471 \pm 0.041$, respectively.

As shown in Table 2, no significant associations were found between LC prognosis and age or lymph node detection. Differentiation, TN, clinical stage, and surgical method were associated with survival time, with hazard ratios for risk of death $(95 \% \mathrm{CI})$ of 2.40 (1.15-4.50),
2.17 (1.45-3.25), 2.39 (1.58-3.62), 3.29 (2.10-5.18), respectively.

\section{Eighteen SNPs were associated with clinical outcome in LC patients}

Genotype frequencies for the eighteen potentially relevant SNPs identified are shown in Table 3. We further explored the roles of 18 of these genetic polymorphisms in predicting LC prognosis. Four SNPs (rs1321311, rs2494938, rs9363918, and rs3016539) were associated with OS in the study population as a whole after adjusting for potentially confounding variables (age, differentiation, $\mathrm{TN}$, clinical stages, surgical method, and lymph node detection) and with genotype as an indicator variable.

As shown in Table 4 and Figure 1, HRs were higher for individuals with rs1321311, rs2494938, or rs9363918 polymorphisms. The rs1321311 TG (53 ${ }_{\text {total }}$, $\left.35_{\text {events }}\right)$ genotype (vs. GG) (117 total $\left.65_{\text {events }}\right)$, rs2494938 AA $\left(8_{\text {total }}, 7\right.$ events $)$ genotype (vs. GG) $\left(91_{\text {total }}, 50_{\text {events }}\right)$, and rs9363918 TG ( $\left.41_{\text {total }}, 34_{\text {events }}\right)$ genotype (vs. GG) (126 $6_{\text {total }}$, $\left.65_{\text {events }}\right)$ were associated with shorter OS (adjusted HR = $1.64,95 \%$ confidence interval $=1.07-2.51, p=0.022$; adjusted $\mathrm{HR}=2.85,95 \% \mathrm{CI}=1.26-6.46, p=0.12$; adjusted $\mathrm{HR}=1.78,95 \% \mathrm{CI}=1.15-2.74, p=0.009$, respectively). The five year Kaplan-Meier survival rates were $0.524 \pm 0.48$ and $0.339 \pm 0.77$ for patients with rs1321311 G/G and G/A genotypes, $0.52 \pm 0.57,0.43 \pm$ 0.69 , and $0.25 \pm 0.15$ for patients with $\mathrm{rs} 2494938 \mathrm{G} / \mathrm{G}$, $\mathrm{A} / \mathrm{G}$, and $\mathrm{A} / \mathrm{A}$ genotypes, and $0.53 \pm 0.48,0.30 \pm 0.73$, and $0.67 \pm 0.27$ for patients with rs9363918 G/G, T/G, and $\mathrm{T} / \mathrm{T}$ genotypes, respectively. Additionally, HRs were lower for individuals with rs3016539 polymorphisms. The HR for the rs3016539 AG $\left(62_{\text {total }}, 31_{\text {events }}\right)$ genotype (vs. AA) $\left(107_{\text {total }}, 68_{\text {events }}\right)$ was $0.603,95 \% \mathrm{Cl}=0.385-0.944$, $p=0.027$. The five year Kaplan-Meier survival rates for patients with the rs3016539 A/A and A/G genotypes were $0.394 \pm 0.54$ and $0.589 \pm 0.63$, respectively.

\section{DISCUSSION}

Here, we examined whether 18 SNPs located on chromosomes 5, 6, and 7 were associated with survival in a cohort of 170 resected laryngeal carcinoma (LC) patients. The T/G genotype at SNP rs1321311, the A/A genotype at SNP rs2494938, and the T/G genotype at SNP rs9363918 were associated with shorter OS in $\mathrm{LC}$ patients. In contrast, the $\mathrm{A} / \mathrm{G}$ genotype at SNP rs3016539 was associated with longer OS in LC patients. Although this was a preliminary, exploratory study, our results demonstrate for the first time that these gene polymorphisms may serve as independent prognostic markers in post-surgery LC patients. Once our results are confirmed, these SNPs could potentially be used in combination with traditional clinical prognosis factors to improve decisions regarding LSCC treatment. 


\begin{tabular}{|c|c|c|c|}
\hline Variable & & & SD \\
\hline Age (mean) & & 60.75 & 10.08 \\
\hline Median follow-up time (months) & & 38.00 & 30.65 \\
\hline \multirow[t]{2}{*}{ Numbers of events } & survival & 70 & \\
\hline & death & 100 & \\
\hline \multirow{4}{*}{ Cumulative Proportion of patients surviving after the indicated time } & & Estimate & $\mathrm{SE}$ \\
\hline & 1 year & 0.85 & 0.03 \\
\hline & 3 years & 0.57 & 0.04 \\
\hline & 5 years & 0.47 & 0.04 \\
\hline Median survival time & & 48.00 & 9.56 \\
\hline
\end{tabular}

Table 2: Patient, treatment, and follow-up information

\begin{tabular}{|c|c|c|c|c|c|c|c|c|c|}
\hline & \multirow{3}{*}{ Patient characteristic } & \multirow{3}{*}{ No. $(\%)$} & \multirow{3}{*}{ No. of deaths } & \multirow{3}{*}{$\begin{array}{c}\text { Survival times } \\
\text { Median } \pm \text { SE }\end{array}$} & \multicolumn{3}{|c|}{$\begin{array}{l}\text { Cumulative proportion of patients } \\
\text { surviving after the indicated time }\end{array}$} & \multirow{3}{*}{$P$} & \multirow{3}{*}{ HR $(95.0 \% \mathrm{CI})$} \\
\hline & & & & & & Estimate $\pm \mathbf{S E}$ & & & \\
\hline & & & & & 1 year & 3 years & 5 years & & \\
\hline \multirow{2}{*}{ Age } & $<60$ & $80(47.1)$ & 47 & $73.00 \pm 11.30$ & $0.88 \pm 0.04$ & $0.597 \pm 0.055$ & $0.49 \pm 0.06$ & & \\
\hline & $\geq 60$ & $90(52.9)$ & 53 & $30.00 \pm 4.43$ & $0.82 \pm 0.04$ & $0.574 \pm 0.053$ & $0.45 \pm 0.06$ & 1.161 & $0.78(1.72 \sim 0.46)$ \\
\hline \multirow{3}{*}{ Differentiation } & well differentiated & $31(18.2)$ & 18 & $71.00 \pm 28.29$ & $0.87 \pm 0.06$ & $0.541 \pm 0.091$ & $0.50 \pm 0.09$ & $0.011 *$ & \\
\hline & moderately differentiated & $125(73.5)$ & 70 & $59.00 \pm 10.13$ & $0.87 \pm 0.03$ & $0.620 \pm 0.044$ & $0.49 \pm 0.05$ & 0.794 & $0.93(0.56 \sim 1.57)$ \\
\hline & poorly differentiated & $14(8.2)$ & 12 & $15.00 \pm 9.35$ & $0.57 \pm 0.13$ & $0.214 \pm 0.110$ & $0.21 \pm 0.11$ & $0.020 *$ & $2.40(1.15 \sim 5.00)$ \\
\hline \multirow{6}{*}{ Clinical stages } & T1-T2 & $102(60.0)$ & 52 & $77.00 \pm 10.62$ & $0.91 \pm 0.03$ & $0.663 \pm 0.047$ & $0.59 \pm 0.05$ & & \\
\hline & $\mathrm{T} 3-\mathrm{T} 4$ & $68(40.0)$ & 48 & $32.00 \pm 4.97$ & $0.75 \pm 0.05$ & $0.437 \pm 0.061$ & $0.30 \pm 0.06$ & $0.000 *$ & $2.17(1.45 \sim 3.25)$ \\
\hline & No & $116(68.3)$ & 61 & $71.00 \pm 11.59$ & $0.90 \pm 0.03$ & $0.670 \pm 0.044$ & $0.67 \pm 0.04$ & & \\
\hline & N1-N2 & $54(31.8)$ & 39 & $26.00 \pm 6.08$ & $0.74 \pm 0.06$ & $0.361 \pm 0.067$ & $0.30 \pm 0.07$ & $0.000 *$ & $2.39(1.58 \sim 3.62)$ \\
\hline & I-II & $73(42.9)$ & 30 & $98.00 \pm 15.57$ & $0.97 \pm 0.02$ & $0.763 \pm 0.050$ & $0.69 \pm 0.06$ & & \\
\hline & III-IV & $97(57.1)$ & 70 & $32.00 \pm 3.98$ & $0.753 \pm 0.04$ & $0.429 \pm 0.051$ & $0.30 \pm 0.05$ & $0.000 *$ & $3.30(2.10 \sim 5.18)$ \\
\hline \multirow{4}{*}{ Treatment } & Partial laryngectomy & $104(61.2)$ & 50 & $73.00 \pm 11.30$ & $0.904 \pm 0.03$ & $0.678 \pm 0.046$ & $0.58 \pm 0.05$ & & \\
\hline & Total laryngectomy & $66(38.8)$ & 50 & $30.00 \pm 4.43$ & $0.76 \pm 0.05$ & $0.405 \pm 0.061$ & $0.30 \pm 0.06$ & $0.000 *$ & $2.35(1.58 \sim 3.49)$ \\
\hline & cervical lymph node dissection & $37(21.8)$ & 19 & & $0.270 \pm 0.06$ & $0.47 \pm 0.09$ & $0.47 \pm 0.09$ & & \\
\hline & no cervical lymph node dissection & $133(78.2)$ & 81 & $56.00 \pm 10.04$ & $0.89 \pm 0.03$ & $0.60 \pm 0.04$ & $0.48 \pm 0.05$ & 0.19 & $0.71(0.43 \sim 1.19)$ \\
\hline
\end{tabular}

Note: $* P<0.05$

The most common of the four OS-related SNPs, rs 2494938, is located at 6p21.1 in an intron region of $L R F N 2$, a member of the synaptic adhesion-like molecules (SALMs) family that interacts with the N-methyl D-aspartate (NMDA) receptor NR1 subunit through its extracellular or TM1 domains. This may at least partially explain the association between rs2494938 SNP and shorter survival in LC patients. Recent studies indicate that LRFN2 is able to subvert hematopoietic differentiation to increase erythropoiesis, and may also be involved in the regulation of colony forming units and fibroblasts. Additionally, the minor alleles of rs 2494938 are associated with an increased risk of lung cancer, non-cardia gastric cancer, and esophageal squamous-cell carcinoma in Han Chinese patients [13]. Wang et al. also found that rs2494938 SNP was associated with an increased risk of head and neck cancer in a Chinese Han population [14]. We found that two additional SNPs, at rs1321311 and rs9363918, were also associated with shorter survival in LC patients.
We also found that the rs1321311 TT genotype was associated with shorter survival in LC patients. The rs1321311 SNP, located at 6p21, is in linkage disequilibrium with a region that encompasses the CDKN1A gene, which encodes a potent cyclin-dependent kinase inhibitor that binds to and inhibits the activity of cyclin-CDK2 or -CDK 4 complexes. CDKN1A regulates cell cycle progression at the G1 stage, and its expression is tightly controlled by the tumor suppressor protein $\mathrm{p} 53$; CDKN1A thus mediates p53-dependent cell cycle G1 phase arrest in response to a variety of stress stimuli [10]. Several reports indicate that CDKN1A, which is also associated with increased risk of esophageal cancer [15] and colorectal cancer [16], may be a useful predictor and target for cancer treatments involving cell cycle alteration.

The rs9363918 SNP is located upstream of the BAI3 gene at chromosome 6q12. BAI3 may be involved in the inhibition of angiogenesis and glioblastoma development, and is associated with the development of brain and esophageal carcinoma and lung tumors [17-19]. The 
Table 3: Candidate SNPs

\begin{tabular}{|c|c|c|c|c|c|c|}
\hline SNP & Chromosome & Position & Band & Alleles A/B & Gene(s) & Role \\
\hline rs401681 & 5 & 1322087 & $5 p 15.33$ & $\mathrm{~T} / \mathrm{C}$ & CLPTM1L & Intron \\
\hline rs6879627 & 5 & 2109901 & $5 p 15.33$ & $\mathrm{~T} / \mathrm{C}$ & & \\
\hline rs13361707 & 5 & 40791884 & $5 p 13.1$ & $\mathrm{C} / \mathrm{T}$ & PRKAA1 & Intron \\
\hline rs367615 & 5 & 108948937 & $5 q 21.3$ & $\mathrm{~T} / \mathrm{C}$ & & \\
\hline rs647161 & 5 & 134499092 & $5 q 31.1$ & $\mathrm{~A} / \mathrm{C}$ & & \\
\hline rs9502893 & 6 & 1340189 & $6 \mathrm{p} 25.3$ & $\mathrm{C} / \mathrm{T}$ & & \\
\hline rs 1321311 & 6 & 36622900 & $6 \mathrm{p} 21.2$ & $\mathrm{~T} / \mathrm{G}$ & $S R S F 3-C D K N 1 A$ & \\
\hline rs2494938 & 6 & 40536128 & $6 \mathrm{p} 21.1$ & $\mathrm{~A} / \mathrm{G}$ & LRFN2 & Intron \\
\hline rs10484761 & 6 & 40802261 & $6 \mathrm{p} 21.1$ & $\mathrm{G} / \mathrm{A}$ & & \\
\hline rs9363918 & 6 & 69142008 & $6 q 12$ & $\mathrm{~T} / \mathrm{G}$ & & \\
\hline rs2057314 & 6 & 117819357 & $6 q 22.1$ & $\mathrm{C} / \mathrm{T}$ & $D C B L D 1$ & Intron \\
\hline rs4269383 & 6 & 156197502 & $6 q 25.3$ & $\mathrm{~A} / \mathrm{G}$ & & \\
\hline rs9365723 & 6 & 158435572 & $6 q 25.3$ & $\mathrm{G} / \mathrm{A}$ & SYNJ2 & Intron \\
\hline rs7758229 & 6 & 160840252 & $6 q 25.3$ & $\mathrm{~T} / \mathrm{G}$ & $S L C 22 A 3$ & Intron \\
\hline rs3016539 & 6 & 162236075 & $6 q 26$ & $\mathrm{G} / \mathrm{A}$ & PARK2 & Intron \\
\hline rs2285947 & 7 & 21584088 & $7 \mathrm{p} 15.3$ & $\mathrm{~A} / \mathrm{G}$ & DNAH11 & Intron \\
\hline rs39453 & 7 & 25133849 & $7 \mathrm{p} 15.3$ & $\mathrm{C} / \mathrm{T}$ & & \\
\hline rs10953615 & 7 & 109152711 & $7 q 31.1$ & $\mathrm{G} / \mathrm{A}$ & & \\
\hline
\end{tabular}

Abbreviations: SNP, Single-nucleotide polymorphism.

Table 4: Genetic polymorphisms and prognosis analysis

\begin{tabular}{|c|c|c|c|c|c|c|c|c|c|c|c|}
\hline \multirow[t]{2}{*}{ SNP_ID } & \multirow[t]{2}{*}{ Alleles $\mathbf{A}^{\mathrm{a}} / \mathbf{B}$} & \multirow[t]{2}{*}{$N$} & \multirow[t]{2}{*}{$\begin{array}{l}N \text { of } \\
\text { events }\end{array}$} & \multirow[t]{2}{*}{$\begin{array}{l}\text { Median survival } \\
\text { times }( \pm \mathrm{SE})\end{array}$} & \multicolumn{3}{|c|}{$\begin{array}{l}\text { Cumulative proportion of patients } \\
\text { surviving after the indicated time } \\
\text { (Estimate } \pm S E \text { ) }\end{array}$} & \multirow[t]{2}{*}{$P^{a}$} & \multirow[t]{2}{*}{$\begin{array}{l}\text { Crude HR } \\
(95.0 \% \text { CI })\end{array}$} & \multirow[t]{2}{*}{$P^{\mathrm{b}}$} & \multirow[t]{2}{*}{$\begin{array}{l}\text { Adjusted HR } \\
(95.0 \% \mathrm{CI})\end{array}$} \\
\hline & & & & & 1 year & 3 years & 5years & & & & \\
\hline \multirow[t]{3}{*}{ rs1321311 } & $\mathrm{G} / \mathrm{G}$ & 117 & 65 & & $0.89 \pm 0.03$ & $0.61 \pm 0.05$ & $0.52 \pm 0.05$ & & & & \\
\hline & $\mathrm{T} / \mathrm{G}$ & 53 & 35 & $62.00 \pm 13.26$ & $0.76 \pm 0.06$ & $0.49 \pm 0.07$ & $0.34 \pm 0.08$ & 0.027 & $1.60(1.05 \sim 2.43)$ & $0.022^{*}$ & $1.64(1.07 \sim 2.51)$ \\
\hline & $\mathrm{T} / \mathrm{T}$ & 0 & & & & & & & & & \\
\hline \multirow[t]{3}{*}{ rs2494938 } & $\mathrm{G} / \mathrm{G}$ & 91 & 50 & $62.00 \pm 14.65$ & $0.82 \pm 0.04$ & $0.60 \pm 0.05$ & $0.52 \pm 0.06$ & 0.066 & & 0.038 & \\
\hline & $\mathrm{A} / \mathrm{G}$ & 58 & 36 & $44.00 \pm 6.73$ & $0.52 \pm 0.06$ & $0.59 \pm 0.07$ & $0.43 \pm 0.07$ & 0.663 & $1.10(0.72 \sim 1.69)$ & 0.952 & $1.01(0.65 \sim 1.57)$ \\
\hline & $\mathrm{A} / \mathrm{A}$ & 8 & 7 & $14.00 \pm 4.24$ & $0.63 \pm 0.17$ & $0.25 \pm 0.15$ & $0.25 \pm 0.15$ & 0.020 & $2.58(1.16 \sim 5.72)$ & $0.012^{*}$ & $2.85(1.26 \sim 6.46)$ \\
\hline \multirow[t]{3}{*}{ rs9363918 } & $\mathrm{G} / \mathrm{G}$ & 126 & 65 & $71.00 \pm 13.39$ & $0.86 \pm 0.03$ & $0.62 \pm 0.04$ & $0.53 \pm 0.05$ & 0.014 & & 0.025 & \\
\hline & $\mathrm{T} / \mathrm{G}$ & 41 & 34 & $35.00 \pm 2.46$ & $0.81 \pm 0.06$ & $0.43 \pm 0.08$ & $0.30 \pm 0.07$ & 0.006 & $1.80(1.19 \sim 2.73)$ & $0.009^{*}$ & $1.78(1.15 \sim 2.74)$ \\
\hline & $\mathrm{T} / \mathrm{T}$ & 3 & 1 & & & $0.67 \pm 0.27$ & $0.67 \pm 0.27$ & 0.474 & $0.48(0.07 \sim 3.55)$ & 0.515 & $0.51(0.07 \sim 3.83)$ \\
\hline \multirow[t]{3}{*}{ rs3016539 } & $\mathrm{A} / \mathrm{A}$ & 107 & 68 & 44.00 & $0.81 \pm 0.04$ & $0.54 \pm 0.05$ & $0.39 \pm 0.05$ & & & & \\
\hline & $\mathrm{A} / \mathrm{G}$ & 62 & 31 & 85.00 & $0.92 \pm 0.04$ & $0.64 \pm 0.06$ & $0.59 \pm 0.06$ & 0.015 & $0.59(0.38 \sim 0.90)$ & $0.027^{*}$ & $0.60(0.39 \sim 0.94)$ \\
\hline & $\mathrm{G} / \mathrm{G}$ & 0 & & & & & & & & & \\
\hline
\end{tabular}

Abbreviations: SNP, Single-nucleotide polymorphism; CI, confidence interval; HR, hazard ratio; Adjusted HR: adjusted by age, differentiation, clinical stage, and treatment. ${ }^{*} p<0.05$.

rs3016539 SNP is located in the PARK2 gene, which is one of the largest human genes and codes for the protein parkin. Parkin tags damaged or unneeded proteins with ubiquitin molecules, marking them for degradation [20]. More than 200 PARK2 gene mutations have been identified, and they contribute to Parkinson's disease and several forms of human cancer, including glioblastoma, colorectal cancer, lung cancer, ovarian cancer, breast cancer, renal cancer, and sporadic colorectal cancer. Because parkin is thought to act as a tumor suppressor, reduced parkin function might contribute to uncontrolled cell growth and division, promoting tumor formation. Bartek and Hodny reported that PARK2 expression regulates cyclin-dependent kinases to ensure coordinated cell cycle progression and guard against tumorigenesis [21]. PARK2 is an important tumor suppressor in many different cancers [22, 23], and PARK2 gene alterations are common in many human malignancies $[18,24]$. PARK2 is also associated with aggressive disease and poor clinical outcomes [25]; PARK2 loss might trigger replication stress and fuel tumor progression by increasing 
genomic instability [25]. In our study, the rs3016539 A/G genotype was associated with longer survival in LC patients; it is possible that this mutation enhances the function of the PARK2 gene.

The patients in this study were enrolled in Xi'an and adjacent areas. Due to the similarities in patient characteristics, this patient cohort may be especially useful for revealing population-specific influences of different SNPs; additionally, most patients continued to participate in the study throughout the follow-up period. Furthermore, because these patients received similar treatments, the confounding effects of different therapies that are common in cancer clinical outcome studies were minimized here. However, the limitations of this study should be considered when interpreting the results.
Although there was sufficient statistical power to identify SNP-related differences in survival, the sample size was relatively small; larger studies should be conducted to confirm these results. In addition, future studies should focus on the mechanisms by which the SNPs identified here influence prognosis in Chinese LC patients.

The novel gene polymorphisms identified here that are associated with clinical outcomes in LC patients may help to improve personalized treatment strategies. Our findings suggest that these genes may be useful when screening for LC in Han Chinese populations and might aid in the discovery of additional molecular mechanisms that contribute to LC. Overall, our results strongly suggest that gene polymorphisms may be an independent prognostic marker in resected LC patients.
A

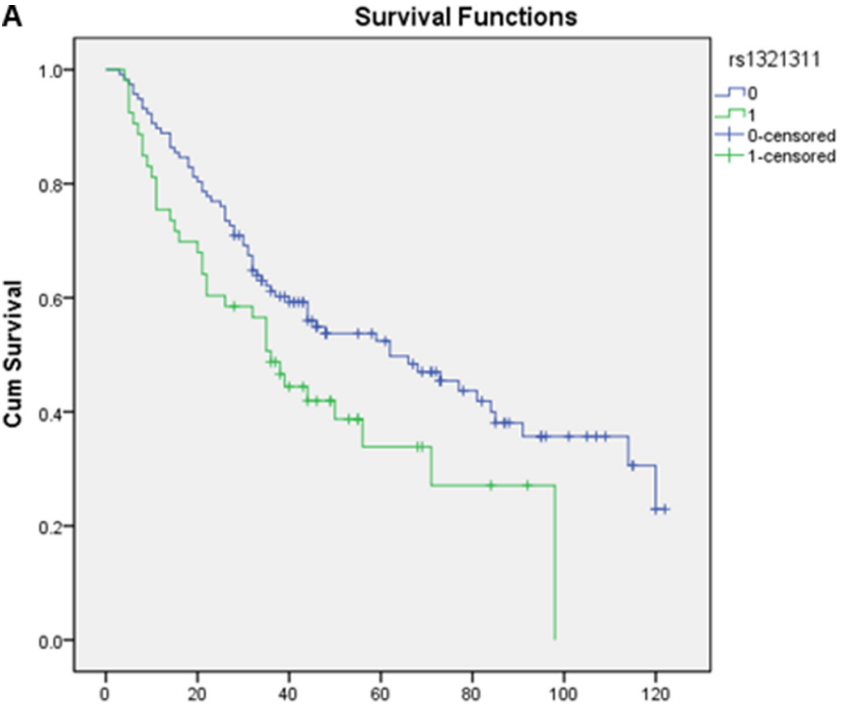

C

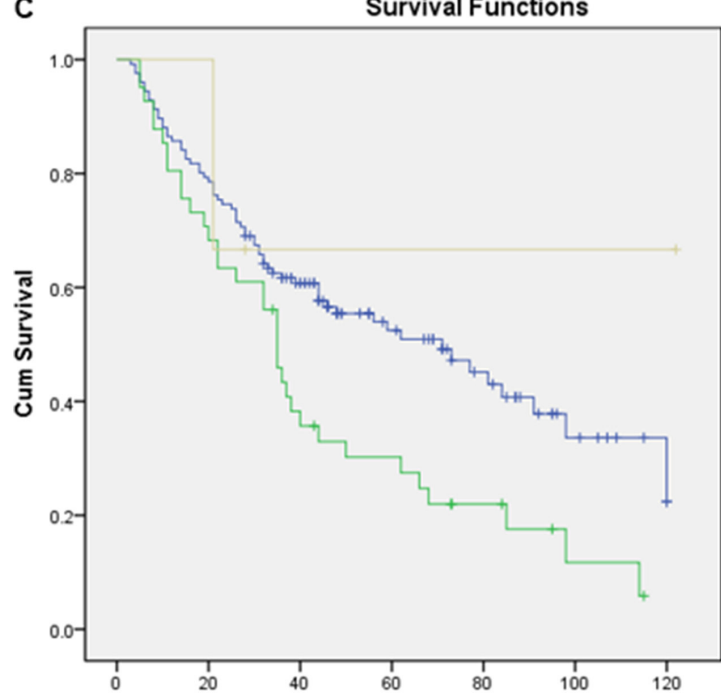

B

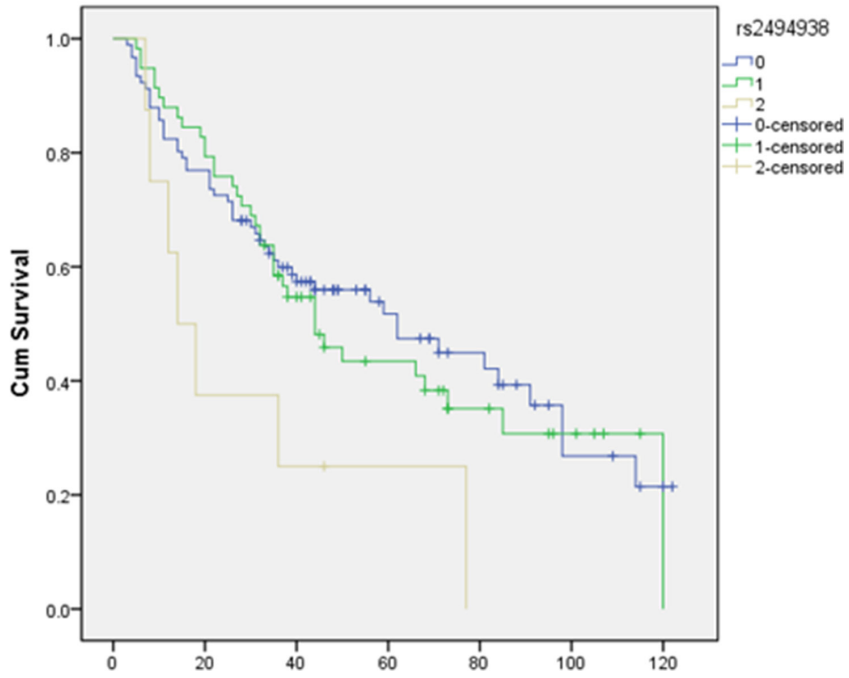

\section{D}

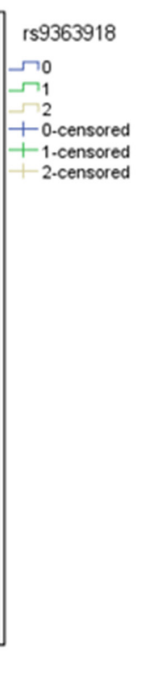

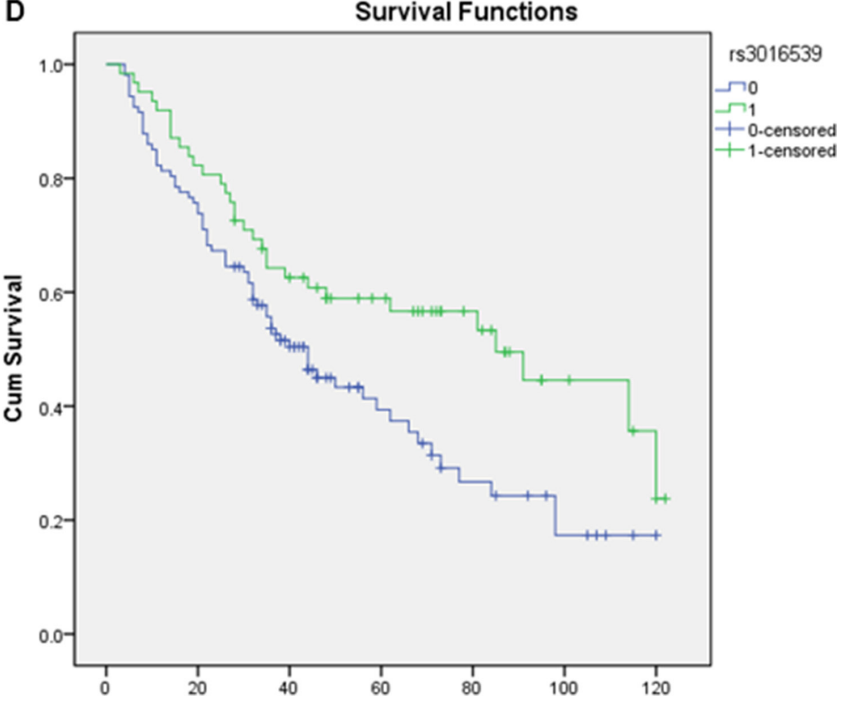

Figure 1: Kaplan-meier plots. 


\section{MATERIALS AND METHODS}

\section{Patient characteristics and treatment}

170 laryngeal carcinoma (stages I to IV) patients who underwent laryngectomy at the First Affiliated Hospital of Xi'an Jiaotong University between January 2002 and April 2013 were included in this study. X-rays, CT, laryngoscopy, examination of laryngeal lesions, local cell smears, and pathology examinations were used for diagnosis. Eligible patients had pathologically-confirmed laryngeal carcinoma without distant metastases (M0). Each tumor was staged according to the 2010 UICC standard staging classification. All patients received surgery within 2 months of diagnosis, and no patient received anticancer treatment before surgery. All patients were Han Chinese males ranging in age from 32 to 82 years. All participants provided informed consent. Patients with recurring disease, or who had secondary laryngeal carcinoma with the primary cancer located elsewhere, were excluded. Patient information (age, sex, race, family history of cancer, and exposure) was recorded using questionnaires, and blood samples were collected for genotyping at the time of study entry.

Patient enrollment ended in 2013 to ensure adequate follow-up times for all participants; follow-ups continued for at least 2 years in all surviving patients. Complete outpatient records were obtained for the 170 patients, who were all treated at the First Affiliated Hospital of Xi'an Jiaotong University; complete genotype data was also available for all 170 patients.

\section{DNA extraction and genotyping}

For each participant, $5 \mathrm{~mL}$ of venous blood was collected in sterile tubes with ethylenediaminetetraacetic acid at the time of enrollment. After centrifugation, samples were stored at $-80^{\circ} \mathrm{C}$ until analysis. Genomic DNA was extracted from blood samples using a GoldMag-Mini Whole Blood Genomic DNA Purification Kit (GoldMag Co. Ltd. Xi'an City, China), and concentration was determined using a Nano Drop 2000 spectrophotometer (Thermo Fisher Scientific, Waltham, MA, USA).

The 18 SNPs analyzed here were identified in LC genome-wide association studies [8-10]. All of the SNPs had minor allele frequencies of $>5 \%$ in the HapMap for the Chinese Han Beijing population. Of these 18 SNPs variants, have been examined in Chinese populations. However, the results of previous genome-wide association studies and studies in Chinese populations are inconsistent. The Multiplexed SNP MassEXTENDED assay was designed using Sequenom MassARRAY Assay Design 3.0 Software (Sequenom Inc, San Diego, CA, USA) [26].

Genotyping of the 18 SNPs was performed using the Sequenom MassARRAY RS1000 system according to the manufacturer's instructions. Internal quality controls and negative controls were used to ensure genotyping accuracy, and $5 \%$ of the samples were randomly selected for repeat runs. Call rates for genotyping ranged from $99.0 \%$ and $99.4 \%$. Two authors independently reviewed all agarose gels, data entry, and statistical analyses.

\section{Survival measurements}

Time to event was defined as the duration of the period from the date of surgery to either event occurrence or the last date of the study (April 7, 2013), whichever came first. All causes of death were included when calculating overall survival (OS). Dates of death were obtained and cross-checked using at least one of the following three methods: Social Security Death Index, the First Affiliated Hospital of Xi' an Jiaotong University tumor registry, and in- or outpatient medical records confirmed by the patient's primary care physician and/or family. Surviving patients were censored using the date of last contact. This date was verified by inpatient and outpatient medical records, and/or confirmation with the patient's primary care physician and/or family.

\section{Statistical analysis}

Overall survival was defined as the time from surgery to death due to laryngeal carcinoma. All genotypes for each SNP were included in the analysis. Hazard ratios (HRs) and 95\% confidence intervals (CIs) were estimated separately for each SNP using the Cox proportional hazard model and after adjusting for age, tumor differentiation, TNM stage, surgical method, and lymph node detection. Cumulative proportions of patients surviving were estimated using the Kaplan-Meier statistical method. The log-rank test was used to evaluate the possible impact of genetic polymorphisms, separately and in combination, on OS. Additional clinical factors were also included in the analysis. $P<0.05$ was considered statistically significant, and all analyses were conducted using the SPSS 17.0 statistical package (SPSS, Chicago, IL).

\section{ACKNOWLEDGMENTS AND FUNDING}

This work was supported by Opening Foundation of Key Laboratory of Resource Biology and Biotechnology in Western China (Northwest University), Ministry of Education

\section{CONFLICTS OF INTEREST}

We declare no conflicts of interest.

\section{REFERENCES}

1. Sato $\mathrm{Y}$, Kosugi S-i, Aizawa N, Ishikawa T, Kano Y, Ichikawa H, Hanyu T, Hirashima K, Bamba T, Wakai T. Risk Factors and Clinical Outcomes of Recurrent Laryngeal Nerve Paralysis After Esophagectomy for Thoracic Esophageal Carcinoma. World J Surg. 2016; 40:129-136. 
2. Zhou J, Li Y, Wei D, Qian Y, Li W, Liu D, Li G, Pan X, Lei D. Overall survival with and without laryngeal function preservation in 580 patients with hypopharyngeal squamous cell carcinoma. Oncol Rep. 2015; 34:3196-3202.

3. Li Z-Q, Zou L, Liu T-R, Yang A-K. Prognostic value of body mass index before treatment for laryngeal squamous cell carcinoma. Cancer Biology Medicine. 2015; 12:394-400.

4. Young RJ, Urban D, Angel C, Corry J, Lyons B, Vallance N, Kleid S, Iseli TA, Solomon B, Rischin D. Frequency and prognostic significance of p16(INK4A) protein overexpression and transcriptionally active human papillomavirus infection in laryngeal squamous cell carcinoma. Brit J Cancer. 2015; 112:1098-1104.

5. Catela Ivkovic T, Loncar B, Spaventi R, Kapitanovic S. Association of H-ras polymorphisms and susceptibility to sporadic colon cancer. Int J Oncol. 2009; 35:1169-1173.

6. Zhang Y, Jin M, Liu B, Ma X, Yao K, Li Q, Chen K. Association between H-RAS T81C genetic polymorphism and gastrointestinal cancer risk: a population based casecontrol study in China. BMC Cancer. 2008; 8:256.

7. Nigro JM, Baker SJ, Preisinger AC, Jessup JM, Hostetter R, Cleary K, Bigner SH, Davidson N, Baylin S, Devilee P and et al. Mutations in the p53 gene occur in diverse human tumour types. Nature. 1989; 342:705-708.

8. Wei Q, Yu D, Liu M, Wang M, Zhao M, Liu M, Jia W, Ma H, Fang J, Xu W. Genome-wide association study identifies three susceptibility loci for laryngeal squamous cell carcinoma in the Chinese population. Nat Genet. 2014; 46:1110-1114.

9. Wang R, Ma H, Lian M, Yang F, Wang H, Feng L, Fang J. [A preliminary study on genome-wide expression profiling of laryngeal squamous cell carcinoma]. Zhonghua er bi yan hou tou jing wai ke za zhi= Chinese journal of otorhinolaryngology head and neck surgery. 2014; 49:232-235.

10. Yang M, Park JY, Tae K. Genome-wide Evidence of XPC Alteration in Laryngeal Squamous Cell Carcinomas. Asian Pac J Cancer P. 2011; 12:1477-1481.

11. Wen S-x, Tang P-z, Zhang X-m, Zhao D, Guo Y-1, Tan W, Lin D-x. Association between genetic polymorphism in xeroderma pigmentosum $\mathrm{G}$ gene and risks of laryngeal and hypopharyngeal carcinomas. Zhongguo yi xue ke xue yuan xue bao Acta Academiae Medicinae Sinicae. 2006; 28:703-706.

12. Unal M, Tamer L, Ates NA, Akbas Y, Pata YS, Vayisoglu Y, Ercan B, Gorur K, Atik U. Glutathione S-transferase M1, $\mathrm{T} 1$, and $\mathrm{P} 1$ gene polymorphism in laryngeal squamous cell carcinoma. Am J Otolaryng. 2004; 25:318-322.

13. Jin G, Ma H, Wu C, Dai J, Zhang R, Shi Y, Lu J, Miao X, Wang M, Zhou Y, Chen J, Li H, Pan S, et al. Genetic variants at 6 p21.1 and 7 p15.3 are associated with risk of multiple cancers in Han Chinese. Am J Hum Genet. 2012; 91:928-934.

14. Wang R, Zhu L, Zhang Y, Miao L, Ma H, Yuan H, Chen N. Genetic variants at $6 \mathrm{p} 21.1$ are associated with head and neck cancer in Chinese Han population. Cancer Biomark. 2015; 15:27-32.

15. Geng TT, Xun XJ, Li S, Feng T, Wang LP, Jin TB, Hou P. Association of colorectal cancer susceptibility variants with esophageal cancer in a Chinese population. World J Gastroenterol. 2015; 21:6898-6904.

16. Kantor ED, Hutter CM, Minnier J, Berndt SI, Brenner H, Caan BJ, Campbell PT, Carlson CS, Casey G, Chan AT, Chang-Claude J, Chanock SJ, Cotterchio M, et al. Geneenvironment interaction involving recently identified colorectal cancer susceptibility Loci. Cancer Epidemiol Biomarkers Prev. 2014; 23:1824-1833.

17. Bari MF, Brown H, Nicholson AG, Kerr KM, Gosney JR, Wallace WA, Soomro I, Muller S, Peat D, Moore JD. BAI3, CDX2 and VIL1: a panel of three antibodies to distinguish small cell from large cell neuroendocrine lung carcinomas. Histopathology. 2014; 64:547-556.

18. Beroukhim R, Mermel CH, Porter D, Wei G, Raychaudhuri S, Donovan J, Barretina J, Boehm JS, Dobson J, Urashima M, Mc Henry KT, Pinchback RM, Ligon AH, et al. The landscape of somatic copy-number alteration across human cancers. Nature. 2010; 463:899-905.

19. Kee HJ, Ahn KY, Choi KC, Won Song J, Heo T, Jung S, Kim JK, Bae CS, Kim KK. Expression of brain-specific angiogenesis inhibitor 3 (BAI3) in normal brain and implications for BAI3 in ischemia-induced brain angiogenesis and malignant glioma. FEBS Lett. 2004; 569:307-316.

20. Wang J, Zhang B, Yang Z, Zhou L, Geng T, Li H, Fu X, Xue X, Liu M, Tong R, Jin T, Zhang Y. Association of gastrointestinal gland cancer susceptibility loci with esophageal carcinoma among the Chinese Han population: a case-control study. Tumour Biol. 2015.

21. Bartek J, Hodny Z. PARK2 orchestrates cyclins to avoid cancer. Nat Genet. 2014; 46:527-528.

22. Kapitanovic S, Cacev T, Loncar B, Catela Ivkovic T, Krizanac S, Pavelic K. Reduced FHIT expression is associated with tumor progression in sporadic colon adenocarcinoma. Exp Mol Pathol. 2014; 96:92-97.

23. Siprashvili Z, Sozzi G, Barnes LD, McCue P, Robinson AK, Eryomin V, Sard L, Tagliabue E, Greco A, Fusetti L, Schwartz G, Pierotti MA, Croce CM, et al. Replacement of Fhit in cancer cells suppresses tumorigenicity. Proceedings of the National Academy of Sciences of the United States of America. 1997; 94:13771-13776.

24. Gong Y, Zack TI, Morris LG, Lin K, Hukkelhoven E, Raheja R, Tan IL, Turcan S, Veeriah S, Meng S, Viale A, Schumacher SE, Palmedo P, et al. Pan-cancer genetic analysis identifies PARK2 as a master regulator of G1/S cyclins. Nat Genet. 2014; 46:588-594.

25. Veeriah S, Taylor BS, Meng S, Fang F, Yilmaz E, Vivanco I, Janakiraman M, Schultz N, Hanrahan AJ, Pao W, Ladanyi M, Sander C, Heguy A, et al. Somatic mutations of the Parkinson's disease-associated gene PARK2 in glioblastoma and other human malignancies. Nat Genet. 2010; 42:77-82.

26. Gabriel S, Ziaugra L, Tabbaa D. SNP genotyping using the Sequenom MassARRAY iPLEX platform. Current protocols in human genetics / editorial board, Jonathan L Haines [et al]. 2009; Chapter 2:Unit 2.12-Unit 12.12. 